\title{
A geometry interface for gyrokinetic microturbulence investigations in toroidal configurations
}

\author{
P. Xanthopoulos, ${ }^{1}$ W. A. Cooper, ${ }^{2}$ F. Jenko, ${ }^{3}$ Yu. Turkin, ${ }^{1}$ A. Runov,${ }^{1}$ and J. Geiger ${ }^{1}$ \\ ${ }^{1}$ EURATOM Association, Max-Planck-Institut für Plasmaphysik, Wendelsteinstr. 1, \\ D-17491 Greifswald, Germany \\ ${ }^{2}$ Centre de Recherches en Physique des Plasmas, Ecole Polytechnique Fédérale de Lausanne, Station 13, \\ CH-1015 Lausanne, Switzerland \\ ${ }^{3}$ EURATOM Association, Max-Planck-Institut für Plasmaphysik, Boltzmannstr. 2, \\ D-85748 Garching, Germany
}

(Received 29 April 2009; accepted 6 July 2009; published online 6 August 2009)

\begin{abstract}
The GENE/GIST code package is developed for the investigation of plasma microturbulence, suitable for both stellarator and tokamak configurations. The geometry module is able to process typical equilibrium files and create the interface for the gyrokinetic solver. The analytical description of the method for constructing the geometric elements is documented, together with several numerical evaluation tests. As a concrete application of this product, a cross-machine comparison of the anomalous ion heat diffusivity is presented. (C) 2009 American Institute of Physics.
\end{abstract}

[DOI: 10.1063/1.3187907]

\section{INTRODUCTION}

The behavior of anomalous transport, particularly in stellarator devices, constitutes to date one of the most challenging topics in magnetic fusion research. The limited number of existing studies in nonsymmetric geometries has revealed exciting new features of the turbulence dynamics. For instance, Watanabe and co-workers (see Refs. 1 and 2) recently demonstrated that a modified configuration (inward shifted) of the Large Helical Device (see Ref. 3) manifests lower levels of ion temperature gradient (ITG) turbulence, as compared to the standard scenario, in view of the more favorable zonal flow response. In a similar physics context, it was shown for the quasi-isodynamic stellarator Wendelstein 7-X (see Ref. 4) that a coexistence of different modes is plausible in the nonlinear saturation phase (for details, see Ref. 5).

A promising path toward the exploration of (stellarator) microturbulence is to furnish a well established gyrokinetic code with the required geometric elements, starting from an [three-dimensional (3D)] equilibrium calculation. Such an effort would eliminate the need to use analytical models, which have only a restricted range of applicability. Based on this motivation, we present here the newly developed code Geometry Interface for Stellarators and Tokamaks (GIST), which is designated to generate the necessary geometric information. Further, we show how this code is coupled to the nonlinear gyrokinetic code GENE (see, e.g., Refs. 6-8) in its local mode of operation. Hence, we obtain the so-called GENE/GIST code package for conducting gyrokinetic microturbulence studies in toroidal configurations.

In the framework of stellarator or tokamak analysis, GIST reads in a standard variational moments equilibrium code (VMEC; see, e.g., Ref. 9) or equilibrium fitting code (EFIT) equilibrium file and consistently integrates two independent methods, namely, the magnetohydrodynamic (MHD) and the field line tracing (FLT) approaches, in order to construct a field-aligned coordinate system, which is indispensable for gyrokinetic simulations. We would like to underline the following two features:

(1) There is a possibility for constructing the field-aligned system based either on Boozer coordinates (see Ref. 10) via the MHD approach or on cylindrical coordinates via the FLT approach. The former is usually applied in stellarator cases. Here, an arbitrary selection of the position of the flux tube to be simulated is allowed for, in order to perform surface scans (see Sec. V F). However, there exist magnetic topologies, especially in the context of tokamaks, where this setup is no longer functional, e.g., islands and stochastic regions. Then, the FLT method is still able to create the field-aligned system. A third variant has its own merit, especially for tokamak edge studies (see Ref. 11). In particular, we are in the position to create a mixed coordinate system, resembling the Boozer one but employing the cylindrical toroidal angle as parallel coordinate. In overall, GIST is able to cover all families of toroidal topologies with a multitude of parametrizations.

(2) There is a possibility for extensive consistency checks of the geometric elements. These are necessary in the more demanding stellarator cases, and are performed routinely before starting out with the gyrokinetic calculations. They rely on the combination of both MHD and FLT techniques (the latter recruited exclusively for this purpose in stellarators).

In addition, GIST can be effectively used as a numerical differential geometry apparatus. Here, we demonstrate how to calculate the curvature function of a selected magnetic field line on a stellarator - treated as a surface curve - and there from we validate the "bad curvature" component, which is vital for the development and behavior of microturbulence.

The paper is organized as follows: In Sec. II, the MHD approach for the determination of the geometric quantities is 
presented, in the spirit of Ref. 12, while in Sec. III, we present the FLT method. In Sec. IV, several numerical evaluation tests for the geometric elements are performed, in order to ascertain the validity of the gyrokinetic simulations. Finally, in Sec. V, the description of the GENE/GIST code package is to be found, together with a concrete application, namely, a cross-machine comparison in the context of ITG turbulence between the quasisymmetric stellarator National Compact Stellarator Experiment (NCSX; see Ref. 13) and the DIII-D tokamak (see Ref. 14).

\section{GEOMETRIC ELEMENTS GENERATED VIA MHD APPROACH}

In this section, we present in detail the analytical derivation of the geometric elements for gyrokinetic simulations, exploiting the MHD formulation. The implementation is based on Boozer coordinates, hence we employ the mapping module of the global stability code TERPSICHORE (see Ref. 15), which performs the transformation of the VMEC information by an appropriate reconstruction of the equilibrium.

\section{A. Coordinate system and field representation}

For the labeling of the flux surfaces, the dimensionless quantity

$$
s=\frac{\Phi}{\Phi_{\text {edge }}} \in[0,1]
$$

is employed, where $\Phi$ denotes the toroidal magnetic flux (thereafter, all fluxes are rescaled by $2 \pi$ ) and $\Phi_{\text {edge }}$ is the value at the last closed surface. For the parametrization of the 3D space, we will consider the coordinate system $\{s, \theta, \zeta\}$, where $\theta, \zeta$ are the Boozer poloidal and toroidal angles. In this system, the contravariant representation of the magnetic field reads

$$
\boldsymbol{B}=\Phi^{\prime}(s) \boldsymbol{\nabla} s \times \boldsymbol{\nabla} \theta+\Psi^{\prime}(s) \boldsymbol{\nabla} \zeta \times \nabla s,
$$

where $\Psi$ denotes the poloidal magnetic flux. A modification of the Boozer system which respects the magnetic field alignment is

$$
\left\{x_{B}^{1}, x_{B}^{2}, x_{B}^{3}\right\}=\left\{s, q(s)\left(\theta-\theta_{k}\right)-\zeta, \theta-\theta_{k}\right\} .
$$

Here, $q=\Phi^{\prime}(s) / \Psi^{\prime}(s)$ denotes the safety factor and $\theta_{k}$ is a local to the flux surface quantity called "ballooning angle" (see, e.g., Ref. 16), which determines the position of the flux tube along the field line and together the radial wavenumber. Now, the contravariant representation for the magnetic field takes the equivalent form

$$
\boldsymbol{B}=\Psi^{\prime}(s) \boldsymbol{\nabla} s \times \boldsymbol{\nabla}\left[q(s)\left(\theta-\theta_{k}\right)-\zeta\right] .
$$

The feature of the field alignment is mathematically expressed by the Clebsch condition $\boldsymbol{B} \cdot \boldsymbol{\nabla} x_{B}^{i}=0, i=1,2$, and facilitates the numerical treatment of gyrokinetic simulations. Moreover, the parallel coordinate $x_{B}^{3}$ runs in the range $\left[-N_{p} \pi, N_{p} \pi\right]$, where $N_{p}$ is the prescribed number of poloidal turns covered by the flux tube. Note that the Jacobians of the two systems are equal, hence in the following we use for these the common symbol $\sqrt{g_{B}}$.

\section{B. Curvature components}

A major agent in the development and behavior of microinstabilities is the curvature of the magnetic field lines. Here, we derive the two curvature components which are necessary for the gyrokinetic applications.

Starting from the standard expression for the curvature vector (see, e.g., Ref. 17)

$$
\boldsymbol{\kappa}=-\boldsymbol{b} \times(\boldsymbol{\nabla} \times \boldsymbol{b}),
$$

where $\boldsymbol{b}=\boldsymbol{B} / B$, we calculate the product $\boldsymbol{b} \times \boldsymbol{\kappa}$. Using the force balance relation $\boldsymbol{j} \times \boldsymbol{B}=\boldsymbol{\nabla} P$ and Ampere's law $\boldsymbol{j}=\boldsymbol{\nabla}$ $\times \boldsymbol{B}$ [as usual, $P(s)$ denotes the pressure and $\boldsymbol{j}$ the current density], we obtain

$$
\boldsymbol{b} \times \boldsymbol{\kappa}=\frac{1}{B^{3}} \boldsymbol{B} \times \boldsymbol{\nabla}\left(P+B^{2} / 2\right) .
$$

Introducing the perpendicular wavevector

$$
\boldsymbol{k}_{\perp}=\boldsymbol{\nabla}\left[\zeta-q(s)\left(\theta-\theta_{k}\right)\right]=-\nabla x_{B}^{2},
$$

we consider the quantity $\boldsymbol{k}_{\perp} \cdot \boldsymbol{b} \times \boldsymbol{\kappa}$ appearing in the curvature drift frequency. The secular contribution is defined as

$$
\begin{aligned}
\left(\boldsymbol{k}_{\perp} \cdot \boldsymbol{b} \times \boldsymbol{\kappa}\right)_{\mathrm{sec}} & =-q^{\prime}(s)\left(\theta-\theta_{k}\right) \boldsymbol{\nabla} s \cdot \boldsymbol{b} \times \boldsymbol{\kappa} \\
& =q^{\prime}(s)\left(\theta-\theta_{k}\right) \frac{1}{2 B^{3}} \boldsymbol{B} \times \boldsymbol{\nabla} s \cdot \boldsymbol{\nabla} B^{2} .
\end{aligned}
$$

A small digression is proper at this point. We notice that in Boozer coordinates holds

$$
\sqrt{g_{B}} B^{2}=J \Psi^{\prime}(s)-I \Phi^{\prime}(s)
$$

with $J$ and $I$ denoting the toroidal and poloidal current fluxes, respectively. This is a direct consequence of the contravariant and covariant representations of the magnetic field, taking, in particular, the inner product of Eq. (2) with

$$
\boldsymbol{B}=B_{s} \boldsymbol{\nabla} s+J \boldsymbol{\nabla} \theta-I \boldsymbol{\nabla} \zeta .
$$

Here, we replaced the covariant components of the field by the current fluxes via Ampere's law as

$$
B_{\theta}=J \text { and } B_{\zeta}=-I .
$$

For later use, we also note that representation (2) leads immediately to the definitions of the contravariant field components since it may also be written as

$$
\boldsymbol{B}=\frac{\Phi^{\prime}(s)}{\sqrt{g_{B}}} \boldsymbol{e}_{\zeta}+\frac{\Psi^{\prime}(s)}{\sqrt{g_{B}}} \boldsymbol{e}_{\theta},
$$

therefore (of course, $B^{s}=0$ )

$$
B^{\theta}=\Psi^{\prime}(s) / \sqrt{g_{B}} \text { and } B^{\zeta}=\Phi^{\prime}(s) / \sqrt{g_{B}} .
$$

Returning to the calculation of the secular contribution, Eq. (9) becomes the aid of Eq. (10),

$$
\left(\boldsymbol{k}_{\perp} \cdot \boldsymbol{b} \times \boldsymbol{\kappa}\right)_{\mathrm{sec}}=-q^{\prime}(s)\left(\theta-\theta_{k}\right) \frac{1}{2 B \sqrt{g_{B}}} \boldsymbol{B} \times \nabla s \cdot \nabla \sqrt{g_{B}} .
$$

Using the force balance relation, we separately calculate the product $\boldsymbol{B} \times \boldsymbol{\nabla} \cdot \boldsymbol{\nabla} \sqrt{g_{B}}$ as follows: First, 


$$
\boldsymbol{\nabla} \cdot\left(\frac{\boldsymbol{B} \times \boldsymbol{\nabla} P}{B^{2}}\right)=-\boldsymbol{B} \cdot \boldsymbol{\nabla}\left(\frac{\boldsymbol{j} \cdot \boldsymbol{B}}{B^{2}}\right),
$$

where the charge conservation relation $\boldsymbol{\nabla} \cdot \boldsymbol{j}=0$ and Maxwell's law $\boldsymbol{\nabla} \cdot \boldsymbol{B}=0$ were employed. The left hand side of the above equation becomes, also considering Eq. (10),

$$
\begin{aligned}
\boldsymbol{\nabla} \cdot\left(\frac{\boldsymbol{B} \times \boldsymbol{\nabla} P}{B^{2}}\right)= & \frac{P^{\prime}(s)}{\sqrt{g_{B}} B^{2}}\left[\sqrt{g_{B}} \boldsymbol{\nabla} \cdot(\boldsymbol{B} \times \boldsymbol{\nabla} s)\right. \\
& \left.+\boldsymbol{B} \times \boldsymbol{\nabla} s \cdot \boldsymbol{\nabla} \sqrt{g_{B}}\right] .
\end{aligned}
$$

From Ampere's law and $j^{s}=0$, it is inferred that $\boldsymbol{\nabla} \cdot(\boldsymbol{B}$ $\times \nabla s)=0$ so we end up with the required expression

$$
\boldsymbol{B} \times \nabla s \cdot \nabla \sqrt{g_{B}}=-\frac{\sqrt{g_{B}} B^{2}}{P^{\prime}(s)} \boldsymbol{B} \cdot \nabla\left(\frac{\boldsymbol{j} \cdot \boldsymbol{B}}{B^{2}}\right),
$$

where we also used Eq. (16). Finally, the expression for the secular contribution turns into

$$
\left(\boldsymbol{k}_{\perp} \cdot \boldsymbol{b} \times \boldsymbol{\kappa}\right)_{\mathrm{sec}}=\frac{q^{\prime}(s) B}{2 P^{\prime}(s)} \boldsymbol{B} \cdot \boldsymbol{\nabla}\left(\frac{\boldsymbol{j} \cdot \boldsymbol{B}}{B^{2}}\right)\left(\theta-\theta_{k}\right) .
$$

We proceed with the quasiperiodic contribution, defined as

$$
\begin{aligned}
\left(\boldsymbol{k}_{\perp} \cdot \boldsymbol{b} \times \boldsymbol{\kappa}\right)_{\mathrm{qper}} & =(\boldsymbol{\nabla} \zeta-q \boldsymbol{\nabla} \theta) \cdot \boldsymbol{b} \times \boldsymbol{\kappa} \\
& =(\boldsymbol{\nabla} \zeta-q \boldsymbol{\nabla} \theta) \cdot \frac{1}{B^{3}} \boldsymbol{B} \times \boldsymbol{\nabla}\left(P+B^{2} / 2\right) .
\end{aligned}
$$

Using the covariant representation of the magnetic field (11), we get

$$
\begin{aligned}
\left(\boldsymbol{k}_{\perp} \cdot \boldsymbol{b} \times \boldsymbol{\kappa}\right)_{\mathrm{qper}}= & \frac{B_{s}}{B^{3}}(\boldsymbol{\nabla} \zeta \times \nabla s+q \boldsymbol{\nabla} s \times \boldsymbol{\nabla} \theta) \cdot \boldsymbol{\nabla}\left(P+B^{2} / 2\right) \\
& -\frac{J-q I}{B^{3}} \nabla \theta \times \nabla \zeta \cdot \nabla\left(P+B^{2} / 2\right) .
\end{aligned}
$$

Employing, in addition, the contravariant representation of the magnetic field (2), we deduce

$$
\begin{aligned}
\left(\boldsymbol{k}_{\perp} \cdot \boldsymbol{b} \times \boldsymbol{\kappa}\right)_{\mathrm{qper}}= & \frac{B_{s}}{\Psi^{\prime}(s) B^{3}} \boldsymbol{B} \cdot \boldsymbol{\nabla}\left(P+B^{2} / 2\right) \\
& -\frac{J \Psi^{\prime}(s)-I \Phi^{\prime}(s)}{\sqrt{g_{B}} \Psi^{\prime}(s) B^{3}} \partial_{s}\left(P+B^{2} / 2\right)
\end{aligned}
$$

and with the aid of Eq. (10) plus a little manipulation, the above expression is rewritten as

$$
\begin{aligned}
\left(\boldsymbol{k}_{\perp} \cdot \boldsymbol{b} \times \boldsymbol{\kappa}\right)_{\mathrm{qper}}= & -\frac{B}{2 \sqrt{g_{B}} \Psi^{\prime}(s)}\left\{\sqrt{g_{B}} B_{s} \boldsymbol{B} \cdot \boldsymbol{\nabla}\left(1 / B^{2}\right)\right. \\
& +\frac{2 \sqrt{g_{B}} P^{\prime}(s)}{B^{2}}+\frac{1}{B^{2}} \partial_{s}\left[J \Psi^{\prime}(s)-I \Phi^{\prime}(s)\right] \\
& \left.-\partial_{s} \sqrt{g_{B}}\right\} .
\end{aligned}
$$

We additionally write

$$
\begin{aligned}
\partial_{s}\left[J \Psi^{\prime}(s)-I \Phi^{\prime}(s)\right]= & J \Psi^{\prime \prime}(s)-I \Phi^{\prime \prime}(s) \\
& +\left[J^{\prime}(s) \Psi^{\prime}(s)-I^{\prime}(s) \Phi^{\prime}(s)\right] .
\end{aligned}
$$

We now concentrate on the last bracket. Specifically, it holds that

$$
J^{\prime}(s) \Psi^{\prime}(s)-I^{\prime}(s) \Phi^{\prime}(s)=\sqrt{g_{B}} \boldsymbol{B} \cdot \boldsymbol{\nabla} B_{s}-\sqrt{g_{B}} P^{\prime}(s) .
$$

Indeed, starting from the force balance relation, we get

$$
\sqrt{g_{B}}\left(j^{\theta} B^{\zeta}-j^{\zeta} B^{\theta}\right)=P^{\prime}(s) .
$$

Furthermore, the contravariant components of the current density are written as

$$
j^{\theta}=\frac{1}{\sqrt{g_{B}}}\left[I^{\prime}(s)+\partial_{\zeta} B_{s}\right] \text { and } j^{\zeta}=\frac{1}{\sqrt{g_{B}}}\left[J^{\prime}(s)-\partial_{\theta} B_{s}\right] .
$$

Backsubstituting these expressions into Eq. (26), together with Eq. (13), we get to the required result. The final expression for the quasiperiodic part of the curvature term now reads

$$
\begin{aligned}
\left(\boldsymbol{k}_{\perp} \cdot \boldsymbol{b} \times \boldsymbol{\kappa}\right)_{\mathrm{qper}}= & -\frac{B}{2 \sqrt{g_{B}} \Psi^{\prime}(s)}\left\{\sqrt{g_{B}} \boldsymbol{B} \cdot \boldsymbol{\nabla}\left(B_{s} / B^{2}\right)\right. \\
& +\frac{\sqrt{g_{B}} P^{\prime}(s)}{B^{2}}+\frac{1}{B^{2}}\left[J \Psi^{\prime \prime}(s)-I \Phi^{\prime \prime}(s)\right] \\
& \left.-\partial_{s} \sqrt{g_{B}}\right\} .
\end{aligned}
$$

Finally, we extract the following expressions which enter the gyrokinetic Vlasov equation, namely,

$$
\begin{aligned}
\boldsymbol{b} \times \boldsymbol{\kappa} \cdot \nabla s & =-\frac{1}{q^{\prime}(s)\left(\theta-\theta_{k}\right)}\left(\boldsymbol{k}_{\perp} \cdot \boldsymbol{b} \times \boldsymbol{\kappa}\right)_{\mathrm{sec}} \\
& =-\frac{B}{2 P^{\prime}(s)} \boldsymbol{B} \cdot \boldsymbol{\nabla}\left(\frac{\boldsymbol{J} \cdot \boldsymbol{B}}{B^{2}}\right)
\end{aligned}
$$

and

$$
\begin{aligned}
\boldsymbol{b} \times \boldsymbol{\kappa} \cdot \nabla\left[q\left(\theta-\theta_{k}\right)-\zeta\right]= & -\left[\left(\boldsymbol{k}_{\perp} \cdot \boldsymbol{b} \times \boldsymbol{\kappa}\right)_{\mathrm{sec}}\right. \\
& \left.+\left(\boldsymbol{k}_{\perp} \cdot \boldsymbol{b} \times \boldsymbol{\kappa}\right)_{\mathrm{qper}}\right] .
\end{aligned}
$$

It is also useful to isolate the contribution of the inhomogeneity of the magnetic field. To this end, we dot multiply Eq. (6) with the perpendicular wavevector and rewrite it as

$$
\boldsymbol{k}_{\perp} \cdot \boldsymbol{b} \times \boldsymbol{\kappa}=\boldsymbol{k}_{\perp} \cdot \boldsymbol{b} \times \frac{\boldsymbol{\nabla} B}{B}+\frac{1}{B^{3}} \boldsymbol{k}_{\perp} \cdot \boldsymbol{B} \times \boldsymbol{\nabla} P .
$$

Now,

$$
\boldsymbol{k}_{\perp} \cdot \boldsymbol{B} \times \nabla P=-\frac{P^{\prime}(s) B^{2}}{\Psi^{\prime}(s)} .
$$

Here, we used the alternative form of Eq. (4),

$$
\boldsymbol{B}=-\Psi^{\prime}(s) \boldsymbol{\nabla} s \times \boldsymbol{k}_{\perp} .
$$

Thus, Eq. (31) turns into 


$$
\boldsymbol{b} \times \frac{\boldsymbol{\nabla} B}{B} \cdot \boldsymbol{\nabla}\left[q\left(\theta-\theta_{k}\right)-\zeta\right]=-\left[\boldsymbol{k}_{\perp} \cdot \boldsymbol{b} \times \boldsymbol{\kappa}+\frac{P^{\prime}(s)}{B \Psi^{\prime}(s)}\right]
$$

Finally, from Eq. (6) it is evident that

$$
\boldsymbol{b} \times \frac{\boldsymbol{\nabla} B}{B} \cdot \boldsymbol{\nabla} s=\boldsymbol{b} \times \boldsymbol{\kappa} \cdot \nabla s .
$$

\section{Metric components}

The expression for the squared magnitude of the perpendicular wavevector can be cast into the form

$$
\left|\boldsymbol{k}_{\perp}\right|^{2}=\frac{\sqrt{g_{B}} B^{2}}{\Psi^{\prime 2}(s)}\left[C_{p}+C_{s}\left(\theta-\theta_{k}\right)+C_{q}\left(\theta-\theta_{k}\right)^{2}\right],
$$

where the coefficients $C_{p}, C_{s}, C_{q}$ are to be defined in the sequel. Introducing the magnetic field line bending vector

$$
\boldsymbol{h}=\frac{\boldsymbol{B} \times \boldsymbol{k}_{\perp}}{B^{2}},
$$

we have

$$
\left|\boldsymbol{k}_{\perp}\right|^{2}=B^{2}\left(h_{s} h^{s}+h_{\theta} h^{\theta}+h_{\zeta} h^{\zeta}\right)
$$

since from Eq. (33), $\boldsymbol{B} \cdot \boldsymbol{k}_{\perp}=0$.

We set out to calculate the contravariant components of $\boldsymbol{h}$. We have, using Eq. (33),

$$
h^{s}=\frac{1}{\Psi^{\prime}(s)} \text {. }
$$

Next, also taking into account Eq. (12),

$$
h^{\theta}=-\frac{1}{\sqrt{g_{B}} B^{2}}\left[B_{s}-I q^{\prime}(s)\left(\theta-\theta_{k}\right)\right]
$$

and

$$
h^{\zeta}=-\frac{1}{\sqrt{g_{B}} B^{2}}\left[q B_{s}-J q^{\prime}(s)\left(\theta-\theta_{k}\right)\right] .
$$

The covariant components of the bending vector are calculated through the contravariant ones and the covariant metric components. For instance,

$$
h_{s}=g_{s s} h^{s}+g_{s \theta} h^{\theta}+g_{s \zeta} h^{\zeta} .
$$

Therefore, Eq. (38) turns into

$$
\begin{aligned}
\left|\boldsymbol{k}_{\perp}\right|^{2}= & B^{2}\left[g_{s s}\left(h^{s}\right)^{2}+2 g_{s \theta} h^{s} h^{\theta}+2 g_{s \zeta} h^{s} h^{\zeta}+g_{\theta \theta}\left(h^{\theta}\right)^{2}\right. \\
& \left.+2 g_{\theta \zeta} h^{\theta} h^{\zeta}+g_{\zeta \zeta}\left(h^{\zeta}\right)^{2}\right],
\end{aligned}
$$

which, on using expressions (39)-(41), takes the form

$$
\begin{aligned}
\left|\boldsymbol{k}_{\perp}\right|^{2}= & B^{2}\left\{\frac{g_{s s}}{\Psi^{\prime 2}(s)}-\frac{2 g_{s \theta}}{\Psi^{\prime}(s) \sqrt{g_{B}} B^{2}}\left[B_{s}-I q^{\prime}(s)\left(\theta-\theta_{k}\right)\right]\right. \\
& -\frac{2 g_{s \zeta}}{\Psi^{\prime}(s) \sqrt{g_{B}} B^{2}}\left[q B_{s}-J q^{\prime}(s)\left(\theta-\theta_{k}\right)\right] \\
& +\frac{g_{\theta \theta}}{\left(\sqrt{g_{B}} B^{2}\right)^{2}}\left[B_{s}-I q^{\prime}(s)\left(\theta-\theta_{k}\right)\right]^{2} \\
& +\frac{g_{\zeta \zeta}}{\left(\sqrt{g_{B}} B^{2}\right)^{2}}\left[q B_{s}-J q^{\prime}(s)\left(\theta-\theta_{k}\right)\right]^{2} \\
& +\frac{2 g_{\theta \zeta}}{\left(\sqrt{g_{B}} B^{2}\right)^{2}}\left[q B_{s}-J q^{\prime}(s)\left(\theta-\theta_{k}\right)\right] \\
& \left.\times\left[B_{s}-I q^{\prime}(s)\left(\theta-\theta_{k}\right)\right]\right\} .
\end{aligned}
$$

Comparing with Eq. (36), the expressions for the coefficients $C_{p}, C_{s}, C_{q}$ are specified. To this end, we also notice that

$$
\boldsymbol{e}_{\theta}+q \boldsymbol{e}_{\zeta}=\sqrt{g_{B}} \boldsymbol{k}_{\perp} \times \nabla s=\frac{\sqrt{g_{B}}}{\Psi^{\prime}(s)} \boldsymbol{B} .
$$

Therefore,

$$
g_{s \theta}+q g_{s \zeta}=\frac{\sqrt{g_{B}} B_{s}}{\Psi^{\prime}(s)}
$$

and

$$
g_{\theta \theta}+2 q g_{\theta \zeta}+q^{2} g_{\zeta \zeta}=\left(\frac{\sqrt{g_{B}} B}{\Psi^{\prime}(s)}\right)^{2} .
$$

With the aid of the above relations we now have

$$
C_{p}=\frac{g_{s s}}{\sqrt{g_{B}}}-\frac{B_{s}^{2}}{\sqrt{g_{B}} B^{2}} .
$$

The next coefficient is

$$
\begin{aligned}
C_{q} & =\frac{\Psi^{\prime 2}(s)}{\sqrt{g_{B}}} \frac{q^{\prime 2}(s)}{\left(\sqrt{g_{B}} B^{2}\right)^{2}}\left(I^{2} g_{\theta \theta}+2 I J g_{\theta \zeta}+J^{2} g_{\zeta \zeta}\right) \\
& =\frac{\Psi^{\prime 2}(s)}{\sqrt{g_{B}}} \frac{q^{\prime 2}(s)}{\left(\sqrt{g_{B}} B^{2}\right)^{2}}\left(I \boldsymbol{e}_{\theta}+J \boldsymbol{e}_{\zeta}\right) \cdot\left(I \boldsymbol{e}_{\theta}+J \boldsymbol{e}_{\zeta}\right) .
\end{aligned}
$$

The dot product is calculated using Eq. (10),

$$
I \boldsymbol{e}_{\theta}+J \boldsymbol{e}_{\zeta}=I \boldsymbol{e}_{\theta}+\boldsymbol{e}_{\zeta}\left(\frac{\sqrt{g_{B}} B^{2}}{\Psi^{\prime}(s)}+q I\right)=\frac{\sqrt{g_{B}}}{\Psi^{\prime}(s)}\left(I \boldsymbol{B}+B^{2} \boldsymbol{e}_{\zeta}\right),
$$

where we also used Eq. (45). Therefore,

$$
\left(I \boldsymbol{e}_{\theta}+J \boldsymbol{e}_{\zeta}\right) \cdot\left(I \boldsymbol{e}_{\theta}+J \boldsymbol{e}_{\zeta}\right)=\left(\frac{\sqrt{g_{B}}}{\Psi^{\prime}(s)}\right)^{2} B^{2}\left(B^{2} g_{\zeta \zeta}-I^{2}\right) .
$$

On the other hand, from representation (13), we get $\boldsymbol{B} \times \boldsymbol{e}_{\zeta}$ $=\Psi^{\prime}(s) \nabla s$ so that

$$
B^{2} g_{\zeta \zeta}-I^{2}=\Psi^{\prime 2}(s) g^{s s} .
$$

Combining Eqs. (49)-(52), we end up with the final expression for the coefficient 


$$
C_{q}=\frac{\Psi^{\prime 2}(s) q^{\prime 2}(s)}{\sqrt{g_{B}} B^{2}} g^{s s} .
$$

The last coefficient is

$$
\begin{aligned}
C_{s}= & \frac{2 q^{\prime}(s) \Psi^{\prime}(s)}{\left(\sqrt{g_{B}} B\right)^{2}}\left(I g_{s \theta}+J g_{s \zeta}\right)-\frac{2 q^{\prime}(s) \Psi^{\prime 2}(s)}{\left(\sqrt{g_{B}} B\right)^{2}} \frac{B_{s}}{\sqrt{g_{B}} B^{2}} \\
& \times\left[I\left(g_{\theta \theta}+q g_{\theta \zeta}\right)+J\left(g_{\theta \zeta}+q g_{\zeta \zeta}\right)\right] .
\end{aligned}
$$

Here, we calculate the terms involving metric combinations. We have

$$
I g_{s \theta}+J g_{s \zeta}=\frac{\sqrt{g_{B}}}{\Phi^{\prime}(s)}\left(J B_{s}-B^{2} g_{s \theta}\right),
$$

where we used relations (10) and (46). Further, employing Eqs. (12) and (45),

$$
I\left(g_{\theta \theta}+q g_{\theta \zeta}\right)+J\left(g_{\theta \zeta}+q g_{\zeta \zeta}\right)=\frac{\sqrt{g_{B}}}{\Psi^{\prime}(s)}\left(I B_{\theta}+J B_{\zeta}\right)=0 .
$$

Collecting the above results, the final expression for the coefficient reads

$$
C_{s}=\frac{2 q^{\prime}(s)}{q}\left(\frac{J B_{s}}{\sqrt{g_{B}} B^{2}}-\frac{g_{s \theta}}{\sqrt{g_{B}}}\right) .
$$

We are now in the position to construct metric components corresponding to the coordinate system (3). From Eq. (53) we immediately have

$$
g_{B}^{11}=\nabla s \cdot \nabla s=\frac{\sqrt{g_{B}} B^{2}}{q^{\prime 2}(s) \Psi^{\prime 2}(s)} C_{q} .
$$

The next metric component we consider coincides with the square magnitude of the perpendicular wavevector, i.e.,

$$
g_{B}^{22}=\nabla\left[q(s)\left(\theta-\theta_{k}\right)-\zeta\right] \cdot \nabla\left[q(s)\left(\theta-\theta_{k}\right)-\zeta\right]=\left|\boldsymbol{k}_{\perp}\right|^{2} .
$$

As a preparatory task for the computation of the last metric component $g_{B}^{12}$, we compare the expression

$$
\begin{aligned}
\left|\boldsymbol{k}_{\perp}\right|^{2}= & q^{\prime 2}(s) g^{s s}\left(\theta-\theta_{k}\right)^{2}+\left[2 q q^{\prime}(s) g^{s \theta}-2 q^{\prime}(s) g^{s \zeta}\right] \\
& \times\left(\theta-\theta_{k}\right)+g^{\zeta \zeta}-2 q g^{\theta \zeta}+q^{2} g^{\theta \theta}
\end{aligned}
$$

stemming directly from the definition, to the one appearing in Eq. (36). This procedure will provide the expression for the coefficient $C_{s}$ as function of the contravariant metrics. Indeed,

$$
C_{s}=\frac{2 q^{\prime}(s) \Psi^{\prime 2}(s)}{\sqrt{g_{B}} B^{2}}\left(q g^{s \theta}-g^{s \zeta}\right) .
$$

We are now able to extract the remaining metric component, using Eqs. (58) and (61), i.e.,

$$
\begin{aligned}
g_{B}^{12} & =\nabla s \cdot \nabla\left[q\left(\theta-\theta_{k}\right)-\zeta\right] \\
& =\frac{\sqrt{g_{B}} B^{2}}{\Psi^{\prime 2}(s) q^{\prime}(s)}\left[C_{q}\left(\theta-\theta_{k}\right)+\frac{C_{s}}{2}\right] .
\end{aligned}
$$

\section{GEOMETRIC ELEMENTS GENERATED VIA FLT APPROACH}

In this section, we describe the algorithm for the generation of geometric elements via the FLT approach (see also Ref. 18). This method is quite powerful, as it is based on the cylindrical components of the magnetic field, which can be read off from a VMEC or EFIT file or even from a BiotSavart solution, in case the equilibrium information is not available. Nevertheless, the generation of the geometry via FLT within an environment of nested magnetic flux surfaces is only practicable if one restricts to a flux tube whose midpoint lies in the symmetry plane of the device. For tokamak configurations, this turns out not to be a handicap-thanks to axisymmetry - therefore, for these cases, FLT is routinely used (see Ref. 11). However, this is no longer true for stellarator devices, where tubes on the same flux surface may demonstrate different turbulence properties. Then, the FLT method is only utilized for checking the consistency of the geometric elements passed to the gyrokinetic solver.

\section{A. Coordinate system and field representation}

We aim at setting up coordinates $\left\{v^{1}, v^{2}, \tau\right\}$ which are aligned to an arbitrary magnetic field line. To achieve this, we follow a constructive method which identifies $v^{1}$ and $v^{2}$ as magnetic (also known as Clebsch) coordinates (see Ref. 17). These two coordinates determine the line itself, while the third coordinate $\tau$ parametrizes the distance along the line. In this way, the 3D space is appropriately resolved.

Magnetic coordinates can be generated by means of the following idea: We consider the surface $\phi=\phi_{k}$ on which the $r$ and $z$ isolines generate an orthogonal grid. Here, $\{r, \phi, z\}$ denote the usual cylindrical coordinates. Now, each point $\left(r_{k}, z_{k}\right)$ in a certain region of interest can be viewed as the starting point of a magnetic field line, and thus the isolines evolve into magnetic surfaces (not necessarily in the sense of flux surfaces). The surfaces containing the point $\left(r_{k}, z_{k}\right)$ shall be described by the equations $v^{1}(\mathbf{R})=0$ and $v^{2}(\mathbf{R})=0$, respectively, where $\mathbf{R}$ denotes the Cartesian position vector. The magnetic field line passing through the grid point $\left(r_{k}, \phi_{k}, z_{k}\right)$ can then be envisaged as the intersection of these two surfaces. This approach entails the equations

$$
\mathbf{B} \cdot \nabla v^{l}=0, \quad l=1,2 .
$$

In order to complete the parametrization of the field line, we introduce a third coordinate describing the distance along it. Usually, this coordinate is the arc length $\ell$, here however, we select another coordinate, namely, $\tau$, which is related to the arc length through the expression

$$
\ell^{\prime}(\tau)=\frac{B}{B^{\phi}},
$$

which means that $\tau$ is not a physical parameter for the representation of the field line (i.e., the field line is not a unitspeed curve). The reason for this specific choice is attributed to the fact that $\tau$ is an anglelike coordinate, which has a trivial connection to the toroidal angle $\phi$. Indeed, from the equation for the field line one gets 


$$
\frac{d \phi}{d \ell}=\frac{B^{\phi}}{B},
$$

which, combined with Eq. (64), yields $d \tau=d \phi$. Using $\tau(0)$ $=\phi_{k}$ as initial condition, we obtain the expression $\tau=\phi-\phi_{k}$, where $\phi_{k}$ becomes the counterpart of the ballooning angle in real space. In this context, note that

$$
B^{\tau}=\mathbf{B} \cdot \nabla \tau=\mathbf{B} \cdot \nabla \phi=B^{\phi}=\frac{B_{\phi}}{r},
$$

with $B_{\phi}$ as the physical cylindrical component.

\section{B. Metric components}

For convenience, we introduce the notation $\left\{y^{1}, y^{2}, y^{3}\right\}$ $=\{r, z, \phi\}$ and $v^{3}=\tau$. The goal then is to determine the derivatives

$$
C_{j}^{l}=\frac{\partial v^{l}}{\partial y^{j}}, \quad j, l=1,2,3
$$

so that we can apply the transformation for the contravariant metric elements (the subscript $c$ refers to the cylindrical system),

$$
g^{k l}=\sum_{i, j=1}^{3} g_{c}^{i j} C_{i}^{k} C_{j}^{l}, \quad \text { where } g_{c}=\operatorname{diag}\left\{1,1, r^{-2}\right\} .
$$

The first step consists in rewriting Eq. (63) as

$$
\sum_{k=1}^{3} B_{c}^{k} C_{k}^{l}=0, \quad l=1,2
$$

where $B_{c}^{k}, k=1,2,3$ are the contravariant cylindrical components of the magnetic field. Now, we differentiate Eq. (69) with respect to $y^{j}, j=1,2,3$ to obtain

$$
\sum_{k=1}^{3} B_{c}^{k} \frac{\partial C_{j}^{l}}{\partial y^{k}}=-\sum_{k=1}^{3} C_{k}^{l} \frac{\partial B_{c}^{k}}{\partial y^{j}}, \quad l=1,2 .
$$

Here, we have tacitly used the relation

$$
\frac{\partial C_{k}^{l}}{\partial y^{j}}=\frac{\partial C_{j}^{l}}{\partial y^{k}}
$$

which stems from the admissibility constraint, namely, $v^{l}$ $\in C^{2}, \quad l=1,2$. At this stage, we shall transform the system of partial differential equation (70) into a corresponding system of ordinary differential equations by employing the field line equation in cylindrical coordinates. In terms of the coordinate $\tau$, this equation reads, also with the aid of Eq. (64),

$$
\frac{d y^{k}}{d \tau}=\ell^{\prime}(\tau) \frac{B_{c}^{k}}{B}=\frac{B_{c}^{k}}{B^{\phi}}, \quad k=1,2,3 .
$$

Thus, combining Eqs. (70) and (72),

$$
\frac{d}{d \tau} C_{j}^{l}=-\sum_{k=1}^{3} \frac{C_{k}^{l}}{B^{\phi}} \frac{\partial B_{c}^{k}}{\partial y^{j}}, \quad l=1,2, \quad j=1,2,3 .
$$

In addition, we have

$$
C_{1}^{3}=C_{2}^{3}=0 \quad \text { and } \quad C_{3}^{3}=1 .
$$

The existence and uniqueness of solution of the linear system of ordinary differential equations (72) and (73) is established once the six quantities $\left.C_{j}^{i}\right|_{0}, i=1,2$, and $j=1,2,3$ are provided at the initial point. These should be determined in compliance with the selected magnetic coordinates, as shown below.

Within a configuration of nested flux surfaces, we typically consider the system $\{\rho, \nu, \chi\}$, where

$$
\rho=\sqrt{\frac{2 \Phi}{B_{a}}}
$$

[note that factor 2 appears as a result of the rescaling of the toroidal flux $\Phi$ with respect to $2 \pi$, given the original "natural" definition $\rho=\sqrt{\Phi / \pi B_{a}}$. The normalizing magnetic field $B_{a}$ is usually chosen so that $\rho$ at the edge equals the (averaged) minor radius $a$ of the device. Therefore [see also Eq. (1)],

$$
B_{a}=\frac{2 \Phi_{\text {edge }}}{a^{2}} \text { and } \rho=a \sqrt{\frac{\Phi}{\Phi_{\text {edge }}}}=a \sqrt{s} .
$$

Squaring Eq. (75) and taking the derivative with respect to $\rho$, we get

$$
\frac{\Psi^{\prime}(\rho)}{B_{a}}=\frac{\rho}{q} .
$$

Now, the representation of the field line lying on the surface $\rho=\rho_{0}$ takes the form (we attach the subscript " 0 " to the flux functions referring to this surface)

$$
\boldsymbol{B}=B_{a} \boldsymbol{\nabla} \rho \times \boldsymbol{\nabla}\left\{\frac{\rho_{0}}{q_{0}}\left[q\left(\theta-\theta_{k}\right)-\zeta\right]\right\} .
$$

The field-following coordinate is the rescaled angle $\chi$ $=\tau / q_{0}=\left(\phi-\phi_{k}\right) / q_{0}$ which lies in the range $\left[-N_{p} \pi, N_{p} \pi\right]$.

Finally, the coordinate system generated by the tracing approach reads

$$
\left\{x_{T}^{1}, x_{T}^{2}, x_{T}^{3}\right\}=\left\{\rho, \frac{\rho_{0}}{q_{0}}\left[q(\rho)\left(\theta-\theta_{k}\right)-\zeta\right], \chi\right\} .
$$

Note that both magnetic coordinates carry dimensions of length. This feature facilitates the transformation from the cylindrical system $(r, z, \phi)$ to the magnetic $(\rho, \nu, \chi)$.

An important quantity related to the metrics is the Jacobian of the coordinate system (79). This is evaluated via representation (78) as follows:

$$
\begin{aligned}
B^{\chi} & =\boldsymbol{B} \cdot \boldsymbol{\nabla} \chi \\
& =B_{a} \boldsymbol{\nabla} \rho \times \nabla\left\{\rho_{0} / q_{0}\left[q\left(\theta-\theta_{k}\right)-\zeta\right]\right\} \cdot \nabla \chi \\
& =B_{a} \sqrt{g_{T}^{-1}},
\end{aligned}
$$

with

$$
B^{\chi}=\frac{B^{\phi}}{q_{0}}=\frac{B_{\phi}}{q_{0} r} .
$$

Therefore, 


$$
{\sqrt{g_{T}}}_{T}=\frac{B_{a}}{B_{\phi}} q_{0} r .
$$

Now we are in a position to apply the initial conditions. In order to tackle this generally undetermined problem, we select the initial point in the symmetry plane of the magnetic configuration, where $\left.B^{r}\right|_{0}=0$ holds, in other words, the $\rho$ $=$ const surfaces are tangent to the $r=$ const surfaces. This fact, in turn, implies that the vector $\nabla x_{T}^{1}$ is parallel to $\nabla r$, therefore,

$$
\left.\boldsymbol{\nabla} x_{T}^{1}\right|_{0}=\left.\frac{\partial \rho}{\partial r}\right|_{0} \boldsymbol{\nabla} r=\left.C_{1}^{1}\right|_{0} \boldsymbol{\nabla} r,
$$

implying that

$$
\left.C_{2}^{1}\right|_{0}=\left.C_{3}^{1}\right|_{0}=0 \text {. }
$$

Next, we utilize the orthogonality condition at the starting point $\left.\left(\nabla x_{T}^{1} \cdot \nabla x_{T}^{2}\right)\right|_{0}=0$, which turns into

$$
\left.\left(C_{1}^{1} C_{1}^{2}+C_{2}^{1} C_{2}^{2}+C_{3}^{1} C_{3}^{2} / r^{2}\right)\right|_{0}=0,
$$

and using Eq. (84) we obtain $\left.C_{1}^{2}\right|_{0}=0$. In order to obtain the field representation (78), the last two coefficients are postulated as

$$
\left.C_{2}^{2}\right|_{0}=\left.\frac{-B_{\phi}}{C_{1}^{1} B_{a}}\right|_{0}
$$

and

$$
\left.C_{3}^{2}\right|_{0}=\left.\frac{r B_{z}}{C_{1}^{1} B_{a}}\right|_{0} .
$$

We remark that conditions (86) and (87) imply that $\mathbf{B} \cdot \nabla x_{T}^{2}$ $=0$. The numerical implementation of the aforementioned setup is carried out by employing a fifth-order Runge-Kutta scheme with adaptive time step.

\section{CONSISTENCY CHECKS FOR GEOMETRIC ELEMENTS}

In this section, we perform systematic comparisons of the geometric elements generated by the two approaches, as described above. These are necessary and are systematically undertaken, particularly in the case of 3D equilibria, in order to ascertain the reliability of the gyrokinetic simulations.

In the following, we select a flux tube for NCSX, with midpoint lying in the symmetry plane of the device, namely, at $(s, \theta, \zeta)=(0.6,0,0)$, so that FLT is applicable. Moreover, the ballooning angle $\theta_{k}$ is set to zero.

\section{A. Metric components}

We analytically relate the metrics corresponding to the FLT and the MHD coordinate systems. Noting that $\rho=a \sqrt{s}$, we successively have

$$
\begin{aligned}
& g_{T}^{11}=\frac{a^{2}}{4 s_{0}} g_{B}^{11}, \\
& g_{T}^{12}=\nabla \rho \cdot \nabla\left\{\rho_{0} / q_{0}\left[q\left(\theta-\theta_{k}\right)-\zeta\right]=\frac{a^{2}}{2 q_{0}} g_{B}^{12},\right.
\end{aligned}
$$
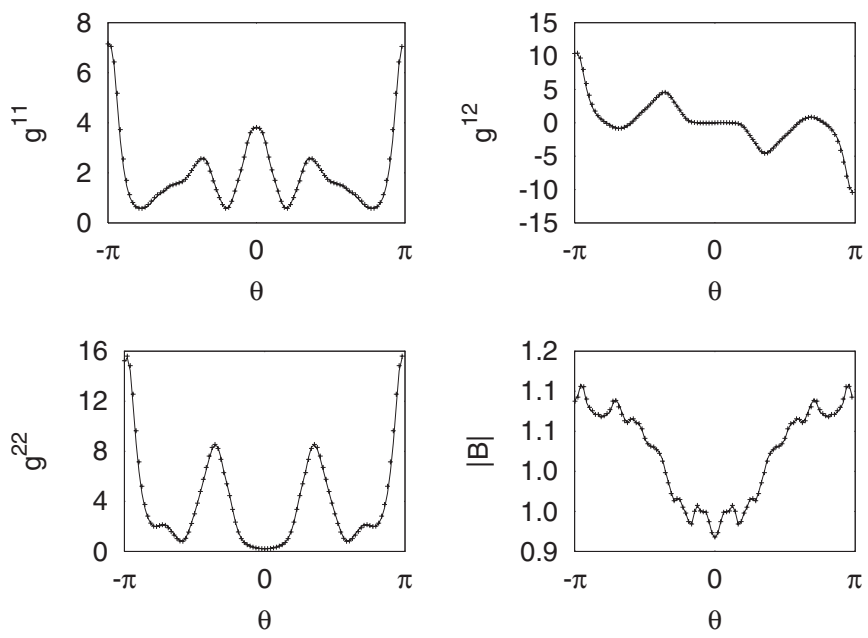

FIG. 1. Comparison of metric components and the magnetic field modulus for NCSX between the FLT (line) and MHD (points) approaches, after the rescalings suggested by Eqs. (88)-(90).

$$
\begin{aligned}
g_{T}^{22} & =\nabla\left\{\rho_{0} / q_{0}\left[q\left(\theta-\theta_{k}\right)-\zeta\right] \cdot \nabla\left\{\rho_{0} / q_{0}\left[q\left(\theta-\theta_{k}\right)-\zeta\right]\right.\right. \\
& =\frac{a^{2} s_{0}}{q_{0}^{2}} g_{B}^{22} .
\end{aligned}
$$

The numerical realization of the above relations is presented in Fig. 1. Here, we mention that the outcome of the FLT method has been remapped onto the poloidal Boozer angle $\theta$, to enable the direct comparison with the MHD approach (the same will be true in following tests, wherever this angle is employed).

\section{B. Field inhomogeneity components}

Here, we relate the expressions for the components of the $\nabla B$ inhomogeneity between the two approaches. For this, we need to use the following identity for the generic scalar fields $A, G$ :

$$
\begin{aligned}
\mathbf{b} \times \nabla A \cdot \nabla G= & \sqrt{g_{T}} b^{\chi}\left[\left(g_{T}^{11} g_{T}^{22}-g_{T}^{12} g_{T}^{12}\right)\left\{\partial_{1} A, \partial_{2} G\right\}\right. \\
& +\left(g_{T}^{11} g_{T}^{23}-g_{T}^{12} g_{T}^{13}\right)\left\{\partial_{1} A, \partial_{3} G\right\} \\
& \left.+\left(g_{T}^{12} g_{T}^{23}-g_{T}^{22} g_{T}^{13}\right)\left\{\partial_{2} A, \partial_{3} G\right\}\right]
\end{aligned}
$$

in terms of the bracket

$$
\left\{\partial_{i} A, \partial_{j} G\right\}=\partial_{i} A \partial_{j} G-\partial_{j} A \partial_{i} G, \quad i, j=1,2,3,
$$

where $\partial_{1}=\partial / \partial x_{T}^{1}$, etc. Thus, we have

$$
\begin{aligned}
\frac{1}{B} \boldsymbol{b} \times \boldsymbol{\nabla} B \cdot \boldsymbol{\nabla} x_{T}^{1}= & -\frac{\sqrt{g_{T}} B^{\chi}}{B^{2}}\left(g_{T}^{11} g_{T}^{22}-g_{T}^{12} g_{T}^{12}\right) \\
& \times\left(\partial_{2} B+\frac{g_{T}^{11} g_{T}^{23}-g_{T}^{12} g_{T}^{13}}{g_{T}^{11} g_{T}^{22}-g_{T}^{12} g_{T}^{12}} \partial_{3} B\right) .
\end{aligned}
$$

From representation (78) we obtain

$$
g_{T}^{11} g_{T}^{22}-g_{T}^{12} g_{T}^{12}=\frac{B^{2}}{B_{a}^{2}} .
$$

As a result, Eq. (93) becomes, also with the aid of Eq. (80), 

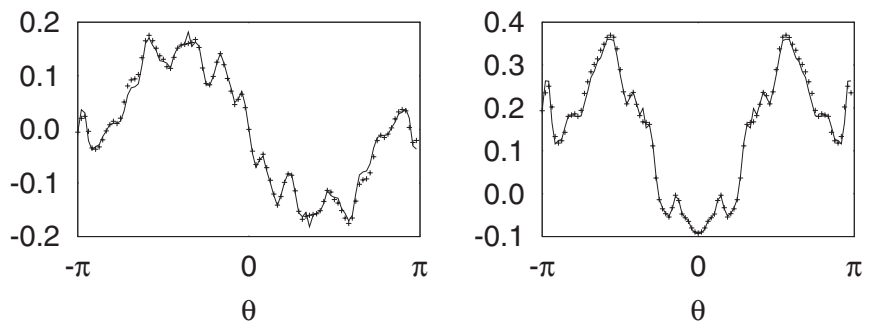

FIG. 2. Numerical validation of Eq. (97) (left) and Eq. (100) (right) for NCSX between FLT (line) and MHD (points) approaches.

$$
\frac{1}{B} \boldsymbol{b} \times \nabla B \cdot \nabla x_{T}^{1}=-\frac{1}{B_{a}}\left(\partial_{2} B+\frac{g_{T}^{11} g_{T}^{23}-g_{T}^{12} g_{T}^{13}}{g_{T}^{11} g_{T}^{22}-g_{T}^{12} g_{T}^{12}} \partial_{3} B\right) .
$$

On the other hand,

$$
\frac{1}{B} \boldsymbol{b} \times \boldsymbol{\nabla} B \cdot \boldsymbol{\nabla} x_{T}^{1}=-\frac{a}{2 \sqrt{s_{0}}} \frac{1}{q_{0}^{\prime}\left(\theta-\theta_{k}\right)}\left(\boldsymbol{k}_{\perp} \cdot \boldsymbol{b} \times \boldsymbol{\kappa}\right)_{\mathrm{sec}}
$$

in view of Eqs. (8) and (35). Thus, it should hold

$$
\begin{gathered}
-\frac{a}{B_{a}}\left(\partial_{2} B+\frac{g_{T}^{11} g_{T}^{23}-g_{T}^{12} g_{T}^{13}}{g_{T}^{11} g_{T}^{22}-g_{T}^{12} g_{T}^{12}} \partial_{3} B\right) \\
=-\frac{a^{2}}{2 \sqrt{s_{0}}} \frac{1}{q_{0}^{\prime}\left(\theta-\theta_{k}\right)}\left(\boldsymbol{k}_{\perp} \cdot \boldsymbol{b} \times \boldsymbol{\kappa}\right)_{\mathrm{sec}} .
\end{gathered}
$$

In the same fashion, we evaluate the other component

$$
\frac{1}{B} \boldsymbol{b} \times \boldsymbol{\nabla} B \cdot \nabla x_{T}^{2}=\frac{1}{B_{a}}\left(\partial_{1} B+\frac{g_{T}^{22} g_{T}^{13}-g_{T}^{12} g_{T}^{23}}{g_{T}^{11} g_{T}^{22}-g_{T}^{12} g_{T}^{12}} \partial_{3} B\right) .
$$

On the other hand, from Eq. (34),

$$
\frac{1}{B} \boldsymbol{b} \times \boldsymbol{\nabla} B \cdot \boldsymbol{\nabla} x_{T}^{2}=-\frac{a \sqrt{s_{0}}}{q_{0}}\left(\boldsymbol{k}_{\perp} \cdot \boldsymbol{b} \times \boldsymbol{\kappa}+\frac{P^{\prime}(s)}{B \Psi^{\prime}(s)}\right) .
$$

Therefore, the final expression relating the two approaches reads

$$
\begin{aligned}
& \frac{a}{B_{a}}\left(\partial_{1} B+\frac{g_{T}^{22} g_{T}^{13}-g_{T}^{12} g_{T}^{23}}{g_{T}^{11} g_{T}^{22}-g_{T}^{12} g_{T}^{12}} \partial_{3} B\right) \\
& =-\frac{a^{2} \sqrt{s_{0}}}{q_{0}}\left(\boldsymbol{k}_{\perp} \cdot \boldsymbol{b} \times \boldsymbol{\kappa}+\frac{P^{\prime}(s)}{B \Psi^{\prime}(s)}\right) .
\end{aligned}
$$

The numerical validation of Eqs. (97) and (100) is presented in Fig. 2.

\section{C. "Bad curvature" component}

This geometric element is of utmost importance for the development and behavior of microturbulence. It is a complicated quantity since it involves both the normal and geodesic curvatures and, for that matter, it is related to the properties of both the surface and the magnetic line. This complexity reflects also on its explicit calculation, as shown below.

\section{Calculation via FLT method}

We consider the magnetic field line as a standard surface curve and we begin by constructing its curvature function. The method can be readily extended for an investigation of the impact of the properties of the field line on the physics.

The transformation rule from the Cartesian system $\{x, y, z\}$ to the (left-handed) cylindrical system $(r, z, \phi)$ reads

$$
x=r(\phi) \cos \phi, \quad y=r(\phi) \sin \phi, \quad z=z(\phi) .
$$

We start with the calculation of the unit tangent vector $\boldsymbol{t}=\dot{\boldsymbol{x}} /\|\dot{\boldsymbol{x}}\|$ (thereafter, the dot signifies the derivative with respect to $\phi)$.

The Cartesian components of this vector are

$$
\begin{aligned}
& t_{x}=\mathcal{A}^{-1}(\dot{r} \cos \phi-r \sin \phi), \\
& t_{y}=\mathcal{A}^{-1}(\dot{r} \sin \phi+r \cos \phi), \\
& t_{z}=\mathcal{A}^{-1} \dot{z},
\end{aligned}
$$

where $\mathcal{A}=\left(\dot{r}^{2}+r^{2}+\dot{z}^{2}\right)^{1 / 2}$. At this stage, it is useful to pass to the physical cylindrical components, i.e.,

$$
\tilde{t}_{r}=\frac{\dot{r}}{\mathcal{A}}, \quad \tilde{t}_{\phi}=\frac{r}{\mathcal{A}}, \quad \tilde{t}_{z}=\frac{\dot{z}}{\mathcal{A}} .
$$

Next, we derive physical cylindrical components of the curvature vector

$$
\boldsymbol{\kappa}=\frac{\ddot{\boldsymbol{x}}\|\dot{\boldsymbol{x}}\|^{2}-(\dot{\boldsymbol{x}} \cdot \ddot{\boldsymbol{x}}) \dot{\boldsymbol{x}}}{\|\dot{\boldsymbol{x}}\|^{4}},
$$

i.e.,

$$
\begin{aligned}
& \widetilde{\kappa}_{r}=\mathcal{A}^{-4}\left[\ddot{r}\left(r^{2}+\dot{z}^{2}\right)-2 r \dot{r}^{2}-r \dot{z}^{2}-r^{3}-\dot{r} \dot{z} \ddot{z}\right], \\
& \widetilde{\kappa}_{\phi}=\mathcal{A}^{-4}\left(2 \dot{r}^{3}+2 \dot{r} \dot{z}^{2}+\dot{r} r^{2}-r \dot{r} \ddot{r}-r \ddot{z} \ddot{z}\right), \\
& \widetilde{\kappa}_{z}=\mathcal{A}^{-4}\left[\ddot{z}\left(\dot{r}^{2}+r^{2}\right)-\dot{z} \ddot{r} \ddot{r}-r \dot{r} \dot{z}\right] .
\end{aligned}
$$

Moreover, one can readily verify that $\widetilde{t}_{r} \widetilde{\kappa}_{r}+\widetilde{t}_{\phi} \widetilde{\kappa}_{\phi}+\widetilde{t}_{z} \widetilde{\kappa}_{z}=0$.

We may now proceed in a straightforward manner to the calculation of the curvature function,

$$
\begin{aligned}
\kappa= & \left(\widetilde{\kappa}_{r}^{2}+\widetilde{\kappa}_{\phi}^{2}+\widetilde{\kappa}_{z}^{2}\right)^{1 / 2} \\
= & \mathcal{A}^{-4}\left\{\left[-r \dot{r} \dot{z}+r^{2} \ddot{z}+\dot{r}(\ddot{r} \ddot{z}-\dot{z} \ddot{r})\right]^{2}\right. \\
& +\left[r^{3}-r^{2} \ddot{r}+r\left(2 \dot{r}^{2}+\dot{z}^{2}\right)+\dot{z}(\dot{r} \ddot{z}-\ddot{r} \dot{z})\right]^{2} \\
& \left.+\left[r^{2} \dot{r}+2 \dot{r}\left(\dot{r}^{2}+\dot{z}^{2}\right)-r(\ddot{r} \ddot{r}+\dot{z} \ddot{z})\right]^{2}\right\}^{1 / 2}
\end{aligned}
$$

by solving the subsystem of Eq. (72).

At this stage, we proceed with the calculation of the bad curvature component. The curvature component in the FLT system reads

$$
\kappa_{1}=\sqrt{g_{T}} \boldsymbol{\kappa} \cdot \boldsymbol{e}^{2} \times \boldsymbol{e}^{3} .
$$

Now, we project the covariant vectors on the physical basis $\left\{\widetilde{\boldsymbol{e}}_{r}, \widetilde{\boldsymbol{e}}_{z}, \widetilde{\boldsymbol{e}}_{\phi}\right\}$ as follows:

$$
\boldsymbol{e}^{2}=C_{1}^{2} \widetilde{\boldsymbol{e}}_{r}+C_{2}^{2} \widetilde{\boldsymbol{e}}_{z}+\frac{C_{3}^{2}}{r} \widetilde{\boldsymbol{e}}_{\phi},
$$




$$
\boldsymbol{e}^{3}=\frac{1}{q_{0} r} \widetilde{\boldsymbol{e}}_{\phi},
$$

where we used Eqs. (67) and (74). Therefore,

$$
\boldsymbol{e}^{2} \times \boldsymbol{e}^{3}=\frac{C_{2}^{2}}{q_{0} r} \widetilde{\boldsymbol{e}}_{r}-\frac{C_{1}^{2}}{q_{0} r} \widetilde{\boldsymbol{e}}_{z}
$$

and finally

$$
\kappa_{1}=\frac{\sqrt{g_{T}}}{q_{0} r}\left(C_{2}^{2} \widetilde{\kappa}_{r}-C_{1}^{2} \widetilde{\kappa}_{z}\right),
$$

where the components $\widetilde{\kappa}_{r}$ and $\widetilde{\kappa}_{z}$ are to be substituted by Eqs. (107) and (109).

\section{Calculation via MHD approach}

The curvature vector is written as

$$
\boldsymbol{\kappa}=\frac{\boldsymbol{\nabla} P}{B^{2}}-\frac{\boldsymbol{B}}{B^{3}} \times(\boldsymbol{B} \times \boldsymbol{\nabla} B)
$$

where Ampere's law and the pressure balance equation were employed. For comparison purposes, we calculate the covariant component $\kappa_{1}=\boldsymbol{\kappa} \cdot \boldsymbol{e}_{1}$ in the FLT system (79). The first term of the above expression gives (on the surface $s=s_{0}$ )

$$
\frac{\nabla P}{B^{2}} \cdot e_{1}=\frac{1}{B^{2}} P^{\prime}\left(\rho_{0}\right)=\frac{2 \sqrt{s_{0}}}{a} \frac{P^{\prime}\left(s_{0}\right)}{B^{2}}
$$

since $\rho=a \sqrt{s}$. The second term becomes

$$
\begin{gathered}
-\frac{\sqrt{g_{T}}}{B^{3}} \boldsymbol{B} \times(\boldsymbol{B} \times \nabla B) \cdot\left(\boldsymbol{e}^{2} \times \boldsymbol{e}^{3}\right) \\
=\frac{\sqrt{g_{T}}}{B^{3}}\left(\boldsymbol{B} \cdot \boldsymbol{e}^{3}\right) \boldsymbol{B} \times \nabla B \cdot \boldsymbol{e}^{2},
\end{gathered}
$$

where we also took into account that $\boldsymbol{B} \cdot \boldsymbol{e}^{2}=0$. Now, $\boldsymbol{B} \cdot \boldsymbol{e}^{3}$ $=B^{\chi}=B_{a}{\sqrt{g_{T}}}^{-1}$, thus

$$
\begin{gathered}
-\frac{\sqrt{g_{T}}}{B^{3}} \boldsymbol{B} \times(\boldsymbol{B} \times \boldsymbol{\nabla} B) \cdot\left(\boldsymbol{e}^{2} \times \boldsymbol{e}^{3}\right) \\
=-\frac{B_{a}}{B} \frac{a \sqrt{s_{0}}}{q_{0}} \boldsymbol{k}_{\perp} \cdot \boldsymbol{b} \times \frac{\boldsymbol{\nabla} B}{B} .
\end{gathered}
$$

In addition, from Eq. (34), we get

$$
\begin{aligned}
- & \frac{\sqrt{g_{T}}}{B^{3}} \boldsymbol{B} \times(\boldsymbol{B} \times \boldsymbol{\nabla} B) \cdot\left(\boldsymbol{e}^{2} \times \boldsymbol{e}^{3}\right) \\
=-\frac{B_{a}}{B} & \frac{a \sqrt{s_{0}}}{q_{0}}\left(\boldsymbol{k}_{\perp} \cdot \boldsymbol{b} \times \boldsymbol{\kappa}+\frac{P^{\prime}\left(s_{0}\right)}{B \Psi^{\prime}\left(s_{0}\right)}\right) .
\end{aligned}
$$

Noting that

$$
\Psi^{\prime}(s)=\frac{B_{a} a^{2}}{2 q},
$$

Eq. (120) becomes

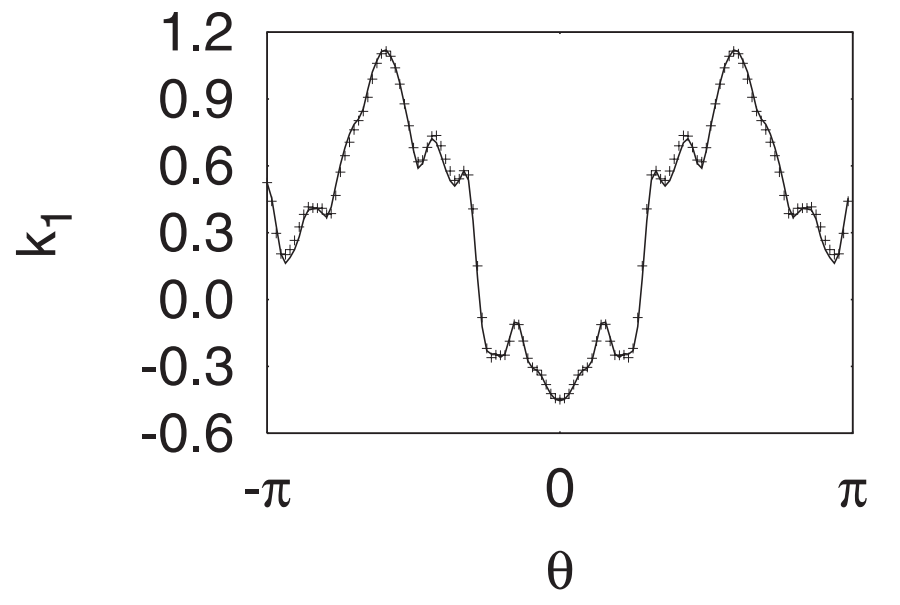

FIG. 3. Numerical comparison of the bad curvature component for NCSX via the MHD (line) and FLT (points) methods.

$$
\begin{aligned}
& -\frac{\sqrt{g_{T}}}{B^{3}} \boldsymbol{B} \times(\boldsymbol{B} \times \nabla B) \cdot\left(\boldsymbol{e}^{2} \times \boldsymbol{e}^{3}\right) \\
& =-\frac{B_{a}}{B} \frac{a \sqrt{s_{0}}}{q_{0}} \boldsymbol{k}_{\perp} \cdot \boldsymbol{b} \times \boldsymbol{\kappa}-\frac{2 \sqrt{s_{0}}}{a} \frac{P^{\prime}\left(s_{0}\right)}{B^{2}} .
\end{aligned}
$$

Gathering Eqs. (117) and (122) we end up with the expression for the bad curvature component (with dimensions of inverse length),

$$
\kappa_{1}=-\frac{B_{a}}{B} \frac{a \sqrt{s_{0}}}{q_{0}} \boldsymbol{k}_{\perp} \cdot \boldsymbol{b} \times \boldsymbol{\kappa} .
$$

Having performed this analysis, we are called to numerically compare expressions (123) and (115) for NCSX. The outcome, manifesting quite good agreement, is presented in Fig. 3.

At this point, we would like to estimate the impact of the minor differences in the geometric elements on the physics output. For that matter, we separately pass the sets of data from the two approaches to the GENE code and consider a linear ITG instability with adiabatic electrons. Then, we perform a scan of the growth rates and frequencies over the binormal wavenumber $k_{y}$. The outcome, presented in Fig. 4, reveals that there is essentially no difference in the gyrokinetic results attributed to the geometry. It is a practical proof that the benchmarking between the FLT and MHD methods is indeed successful.
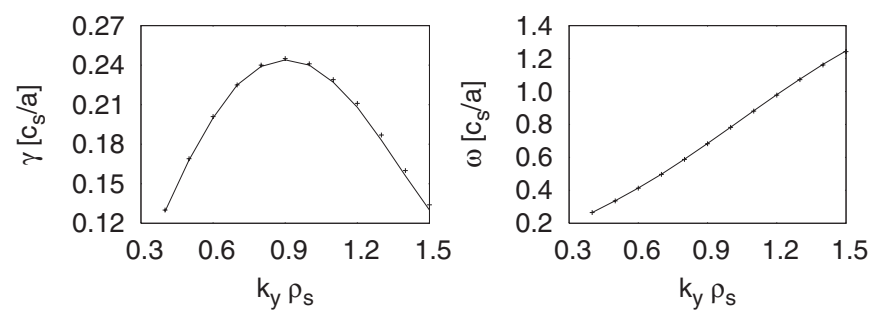

FIG. 4. Scan of growth rates (left) and frequencies (right) over the binormal wavenumber for NCSX using the FLT (line) and MHD (points) settings ( $\rho_{s}=c_{s} / \Omega_{i}, c_{s}$ is the sound speed, $\Omega_{i}$ is the ion Larmor frequency, and $a$ is the minor radius). 


\section{Magnetic shear}

With the term "global magnetic shear" (or simply "shear"), we express the tendency of the flux tube to deform the plane which is spanned by the vectors $\nabla \rho$ and $\nabla \alpha$. Here, we are referring to the generic field-following coordinate system $\left\{\rho, \alpha=\mathcal{D}\left(\rho_{0}\right)[q(\rho) \theta-\zeta], \chi\right\}$, where $\rho$ is the flux label and $\chi(\theta)$ is the parallel coordinate with $\chi( \pm \pi)= \pm \pi$. Moreover, $\mathcal{D}(\rho)$ is a function which takes its form according to the selection of the flux label and $\theta$ and $\zeta$ are any poloidal and toroidal angles, respectively. In other words, starting with a rectangular plane, i.e., $\boldsymbol{\nabla} \rho \cdot \nabla \alpha=0$, the angle between the vectors changes along the flux tube.

In order to quantify the effect of shearing, we provide a simple geometrical picture. The projection of the vector $\nabla \alpha$ on the vector $\nabla \rho$ is

$$
\frac{\nabla \rho \cdot \nabla \alpha}{|\nabla \rho||\nabla \rho|} \nabla \rho=\frac{g^{\rho \alpha}}{g^{\rho \rho}} \nabla \rho .
$$

Then, we define the shear as the average of the rate of change of the prefactor $g^{\rho \alpha} / g^{\rho \rho}$ along the flux tube. Since, in the flux tube approach, this is only dependent on the parallel coordinate $\chi$, we define

$$
\hat{s}=\left\langle\frac{d}{d \chi} \frac{g^{\rho \alpha}}{g^{\rho \rho}}\right\rangle .
$$

We can manipulate this expression as follows:

$$
\begin{aligned}
\frac{g^{\rho \alpha}}{g^{\rho \rho}} & =\frac{\nabla \rho \cdot \nabla\left\{\mathcal{D}\left(\rho_{0}\right)[q(\rho) \theta-\zeta]\right\}}{g^{\rho \rho}} \\
& =\mathcal{D}\left(\rho_{0}\right) q^{\prime}\left(\rho_{0}\right) \theta+\mathcal{D}\left(\rho_{0}\right) \frac{q g^{\rho \theta}-g^{\rho \zeta}}{g^{\rho \rho}},
\end{aligned}
$$

therefore, also writing $d / d \chi=(d / d \theta)\left(1 / \chi^{\prime}(\theta)\right)$,

$$
\frac{d}{d \chi} \frac{g^{\rho \alpha}}{g^{\rho \rho}}=\mathcal{D}\left(\rho_{0}\right) q^{\prime}\left(\rho_{0}\right) \frac{1}{\chi^{\prime}(\theta)}+\mathcal{D}\left(\rho_{0}\right) \tilde{f}^{\prime}(\chi),
$$

where we set the function $\tilde{f}(\chi)=\left(q g^{\rho \theta}-g^{\rho \zeta}\right) / g^{\rho \rho}$, with $\widetilde{f}(-\pi)=\widetilde{f}(\pi)$. Then, taking the flux tube average of Eq. (127), the second term vanishes and we obtain the expression for the shear,

$$
\hat{s}\left(\rho_{0}\right)=\mathcal{D}\left(\rho_{0}\right) q^{\prime}\left(\rho_{0}\right) .
$$

With the term "local magnetic shear" (or simply "local shear") we mean the rate of change in the prefactor $g^{\rho \alpha} / g^{\rho \rho}$ (whose average provides the shear), i.e.,

$$
S_{\mathrm{loc}}=\frac{d}{d \chi} \frac{g^{\rho \alpha}}{g^{\rho \rho}} .
$$

In order to give a more concrete definition, we show that Eq. (129) stems from the vector expression (this coincides with the definition in Ref. 19 but slightly differs from the original one, in Ref. 20)

$$
S_{\mathrm{loc}}=-\sqrt{g} \mathbf{h} \cdot \boldsymbol{\nabla} \times \mathbf{h} \quad \text { with } \mathbf{h}=\frac{1}{g^{\rho \rho}} \mathbf{B}_{N} \times \nabla \rho,
$$

where $\sqrt{g}$ is the Jacobian of the generic field-following coordinate system given by

$$
\sqrt{g}^{-1}=\nabla \rho \times \nabla \alpha \cdot \nabla \chi,
$$

and $\boldsymbol{B}_{N}=\boldsymbol{B} / B_{a}$ is the normalized magnetic field. We have successively

$$
\begin{aligned}
& \mathbf{h}=\nabla \alpha-\frac{g^{\rho \alpha}}{g^{\rho \rho}} \nabla \rho, \\
& \nabla \times \mathbf{h}=\nabla \rho \times \nabla\left(\frac{g^{\rho \alpha}}{g^{\rho \rho}}\right), \\
& \mathbf{h} \cdot \nabla \times \mathbf{h}=-\mathbf{B}_{N} \cdot \nabla\left(\frac{g^{\rho \alpha}}{g^{\rho \rho}}\right) .
\end{aligned}
$$

Further we notice that, along the field line, $\mathbf{B}_{N} \cdot \nabla=\left(B^{\chi} / B_{a}\right)$ $\times(d / d \chi)$ holds, thus

$$
S_{\mathrm{loc}}=\frac{\sqrt{g} B^{\chi}}{B_{a}} \frac{d}{d \chi}\left(\frac{g^{\rho \alpha}}{g^{\rho \rho}}\right) \text {. }
$$

As a final step, we substitute the value of the stream function $\sqrt{g} B^{\chi}=B_{a}$, thus

$$
S_{\mathrm{loc}}=\frac{d}{d \chi}\left(\frac{g^{\rho \alpha}}{g^{\rho \rho}}\right) \text {. }
$$

\section{THE GENE/GIST CODE PACKAGE}

Here we describe the coupling of the GIST code to the gyrokinetic code GENE, by integrating the previously described methods. The outcome of this effort-termed GENE/ GIST code package-proves to be an efficient numerical tool for the investigation of anomalous transport in toroidal configurations. Next, we present the details of the geometric output that the gyrokinetic code handles and we close the section with a concrete application.

\section{A. Coordinate system and field representation}

We begin with the description of the field-aligned coordinate system and the corresponding magnetic field representation. To this aim, we postulate the following dimensionless quantities:

$$
\rho_{N}=\sqrt{s}, \quad \boldsymbol{B}_{N}=\frac{\boldsymbol{B}}{B_{a}}, \quad \boldsymbol{\nabla}_{N}=a \boldsymbol{\nabla}, \quad \Psi_{N}=\frac{\Psi}{a^{2} B_{a}},
$$

the first one being the flux label. Now the field representation becomes

$$
\begin{aligned}
\boldsymbol{B} & =\Psi^{\prime}\left(\rho_{N 0}\right) \boldsymbol{\nabla} \rho_{N} \times \nabla\left[q\left(\rho_{N}\right)\left(\theta-\theta_{k}\right)-\zeta\right] \\
& =B_{a} \nabla_{N} \rho_{N} \times \nabla_{N}\left\{\Psi_{N}^{\prime}\left(\rho_{N 0}\right)\left[q\left(\rho_{N}\right)\left(\theta-\theta_{k}\right)-\zeta\right]\right\}
\end{aligned}
$$

or

$$
\boldsymbol{B}_{N}=\nabla_{N} x^{1} \times \nabla_{N} x^{2}
$$

with

$$
x^{1}=\sqrt{s} \quad \text { and } \quad x^{2}=\Psi_{N}^{\prime}\left(x_{0}^{1}\right)\left[q\left(x^{1}\right)\left(\theta-\theta_{k}\right)-\zeta\right] .
$$

The third coordinate of the system, which parametrizes the distance along the flux tube, can be chosen to be either related to the Boozer system, i.e., 


$$
x_{B}^{3}=\theta-\theta_{k}
$$

or to the cylindrical system, i.e.,

$$
x_{c}^{3}=\left(\phi-\phi_{k}\right) / q_{0},
$$

where, as before, $\phi$ denotes the cylindrical toroidal angle. The explicit choice has an impact on the determination of the Jacobian and the mirror term.

\section{B. Metric components and Jacobian}

The three required metric components for the gyrokinetic simulations are $g^{11}, g^{12}$, and $g^{22}$. We have

$$
g^{11}=\nabla_{N} \rho_{N} \cdot \nabla_{N} \rho_{N}=\frac{a^{2}}{4 s_{0}} g_{B}^{11} .
$$

Next, we deduce the auxiliary relation for the normalized derivative of the poloidal flux

$$
\Psi_{N}^{\prime}\left(x_{0}^{1}\right)=\frac{2 \sqrt{s_{0}}}{a^{2} B_{a}} \frac{\Phi^{\prime}\left(s_{0}\right)}{q_{0}}=\frac{\sqrt{s_{0}}}{q_{0}}
$$

thus,

$$
g^{12}=\nabla_{N} \rho_{N} \cdot \nabla_{N}\left\{\frac{\sqrt{s_{0}}}{q_{0}}\left[q\left(\theta-\theta_{k}\right)-\zeta\right]\right\}=\frac{a^{2}}{2 q_{0}} g_{B}^{12} .
$$

Finally,

$$
g^{22}=\nabla_{N}\left(\frac{\sqrt{s_{0}}}{q_{0}}(q \theta-\zeta)\right) \cdot \nabla_{N}\left(\frac{\sqrt{s_{0}}}{q_{0}}(q \theta-\zeta)\right)=\frac{a^{2} s_{0}}{q_{0}^{2}} g_{B}^{22} .
$$

In addition, it is also necessary to calculate the Jacobian of the system. In the case of selecting $x_{B}^{3}$, this is determined via the MHD method as

$$
\sqrt{g}=\left(\nabla_{N} x^{1} \times \nabla_{N} x^{2} \cdot \nabla_{N} x_{B}^{3}\right)^{-1}=\frac{2 q_{0}}{a^{3}} \sqrt{g_{B}} .
$$

On choosing $x_{c}^{3}$ though, one has to resort to the FLT method to get

$$
\sqrt{g}=\frac{1}{a} \sqrt{g_{T}}
$$

\section{Curvature components}

We analyze the (normalized) curvature drift operator appearing in the gyrokinetic Vlasov equation. Specifically,

$$
\frac{1}{B_{N}} \boldsymbol{b} \times \boldsymbol{\kappa}_{N} \cdot \nabla_{N}=\sum_{i=1}^{2} \frac{1}{B_{N}} \boldsymbol{b} \times \boldsymbol{\kappa}_{N} \cdot \nabla_{N} x^{i} \frac{\partial}{\partial x^{i}}+\mathcal{O}(\boldsymbol{\epsilon}),
$$

where the last term stems from the usual gyrokinetic ordering $\left|\boldsymbol{k}_{\perp}\right| / k_{\|}=\epsilon \ll 1$. GENE admits the following components:

$$
\begin{aligned}
\mathcal{K}_{1} & =\boldsymbol{b} \times \boldsymbol{\kappa}_{N} \cdot \nabla_{N} x^{1} \\
& =\frac{a^{2}}{2 \sqrt{s_{0}}} \boldsymbol{b} \times \boldsymbol{\kappa} \cdot \nabla s \\
& =-\frac{a^{2}}{2 \sqrt{s_{0}}} \frac{1}{q_{0}^{\prime}\left(\theta-\theta_{k}\right)}\left(\boldsymbol{k}_{\perp} \cdot \boldsymbol{b} \times \boldsymbol{\kappa}\right)_{\mathrm{sec}},
\end{aligned}
$$

where we made use of Eq. (29). The second component reads

$$
\begin{aligned}
\mathcal{K}_{2} & =\boldsymbol{b} \times \boldsymbol{\kappa}_{N} \cdot \nabla_{N} x^{2} \\
& =a^{2} \frac{\sqrt{s_{0}}}{q_{0}} \boldsymbol{b} \times \boldsymbol{\kappa} \cdot \nabla\left[q\left(\theta-\theta_{k}\right)-\zeta\right] \\
& =-\frac{a^{2} \sqrt{s_{0}}}{q_{0}} \boldsymbol{k}_{\perp} \cdot \mathbf{b} \times \boldsymbol{\kappa} .
\end{aligned}
$$

\section{Mirror term}

The parallel gradient of the magnetic field determines the mirror force term in the gyrokinetic Vlasov equation (see Ref. 18). As with the Jacobian, we have two possible choices. The first is to assign $x_{B}^{3}$ as parallel coordinate. Then, the relevant expression becomes in normalized form

$$
\left(\nabla_{\|} B\right)_{N}=\boldsymbol{b} \cdot \nabla_{N} B_{N}=\frac{a^{3}}{2 B_{N} q_{0} \sqrt{g_{B}}} \frac{\partial B_{N}}{\partial \theta}
$$

calculated via the MHD method. On the other hand, for $x_{c}^{3}$, the normalized expression in real space reads

$$
\left(\nabla_{\|} B\right)_{N}=\frac{a q_{0}}{B_{N} \sqrt{g_{T}}} \frac{\partial B_{N}}{\partial \phi}
$$

and is calculated via FLT.

\section{E. Magnetic shear}

According to rule (128), the magnetic shear in the GENE/ GIST setup is

$$
\hat{s}=\Psi_{N}^{\prime}\left(x_{0}^{1}\right) q^{\prime}\left(x_{0}^{1}\right)=\frac{2 s_{0}}{q_{0}} q^{\prime}\left(s_{0}\right)
$$

and the expression for the local shear, according to Eq. (133) (for $x^{3}=x_{B}^{3}$ ), is

$$
S_{\mathrm{loc}}=\frac{d}{d \theta}\left(\frac{g^{12}}{g^{11}}\right),
$$

with the metrics as in Eqs. (140) and (142). At this point, we would like to numerically evaluate the relation $\left\langle S_{\text {loc }}\right\rangle=\hat{s}$ for the NCSX case. In Fig. 5, we plot the ratio of the metric components $g^{12} / g^{11}$ as a function of the parallel coordinate for 40 poloidal transits, in order to ascertain a good numerical average. On top of this, we then fit a straight line whose slope has to equal the shear $\hat{s}$. This is because via the fitting procedure, we assume that $\left\langle g^{12} / g^{11}\right\rangle=\lambda \theta$. Thus, the derivative with respect to the parallel coordinate will provide the evaluation of the relation $\hat{s}=\lambda$. The result of this exercise is $\lambda=-0.7594$ to be compared with the value $\hat{s}=-0.7598$, as calculated by Eq. (151). For completeness, in Fig. 6, we plot the local shear profile, according to Eq. (152). 


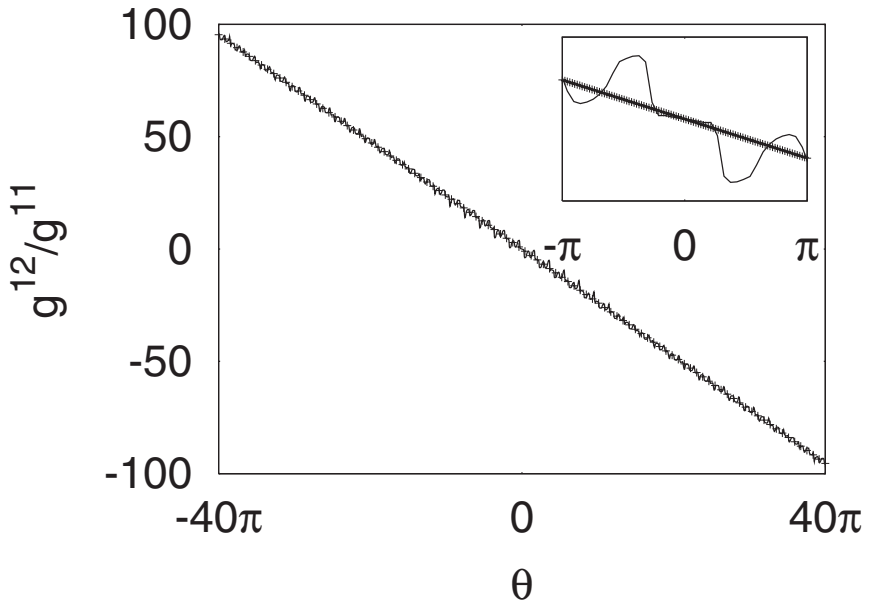

FIG. 5. Numerical evaluation of magnetic shear. The ratio of the metrics (line) is fitted by a straight line (points). The plot in plot presents the same, restricted in the range $\theta \in[-\pi, \pi]$.

\section{F. Application: A cross-machine comparison}

We present for the first time a direct comparison between a stellarator and a tokamak configuration with respect to a typical turbulence property, namely, the ion anomalous heat diffusivity ("figure of merit"), in the context of ITG microturbulence (considering an adiabatic response for the electrons). We define this quantity as

$$
\chi_{i}=-\frac{\rho_{s}^{2} c_{s}}{a\left\langle g^{11}\right\rangle} \frac{\left\langle\hat{Q}_{i}^{1}\right\rangle}{a / T_{i 0} d T_{i 0} / d x^{1}} .
$$

The various symbols have their usual meaning: $a$ denotes the minor radius, $\rho_{s}=c_{s} / \Omega_{i},\left\langle\hat{Q}_{i}^{1}\right\rangle$ is the radial contravariant component of the normalized anomalous ion heat flux (averaged over the flux tube volume and time), and $-1 / T_{i 0} d T_{i 0} / d x^{1}$ is the normalized ITG. Note that via the introduction of the factor $\left\langle g^{11}\right\rangle$, i.e., the flux tube averaged metric, $\chi_{i}$ takes on an invariant form in the sense that it remains unaltered under changes of the radial coordinate.

As a first case for the comparison, we consider the $\operatorname{NCSX}(\mathrm{S} 3)$ equilibrium, selecting a surface with $r / a \approx 0.8$

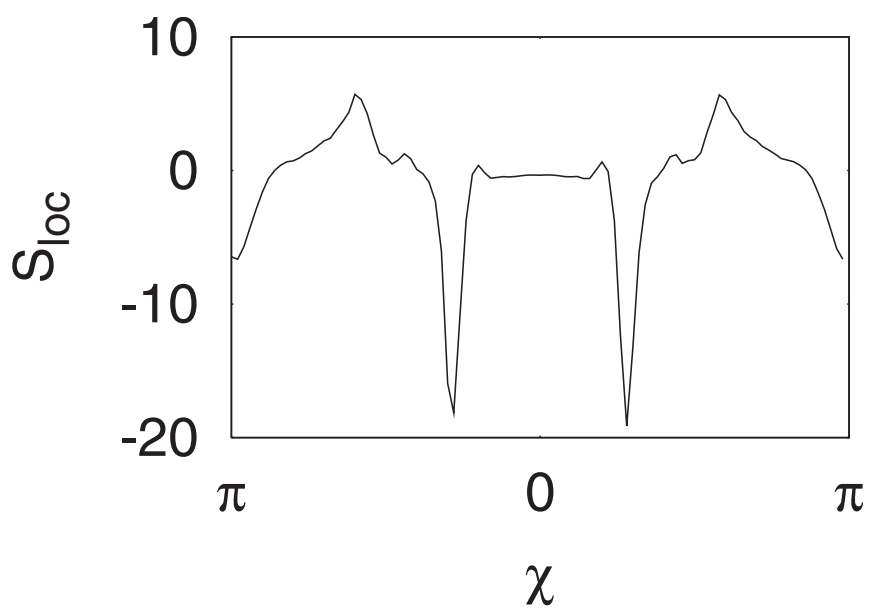

FIG. 6. A typical parallel structure of the local shear for NCSX.

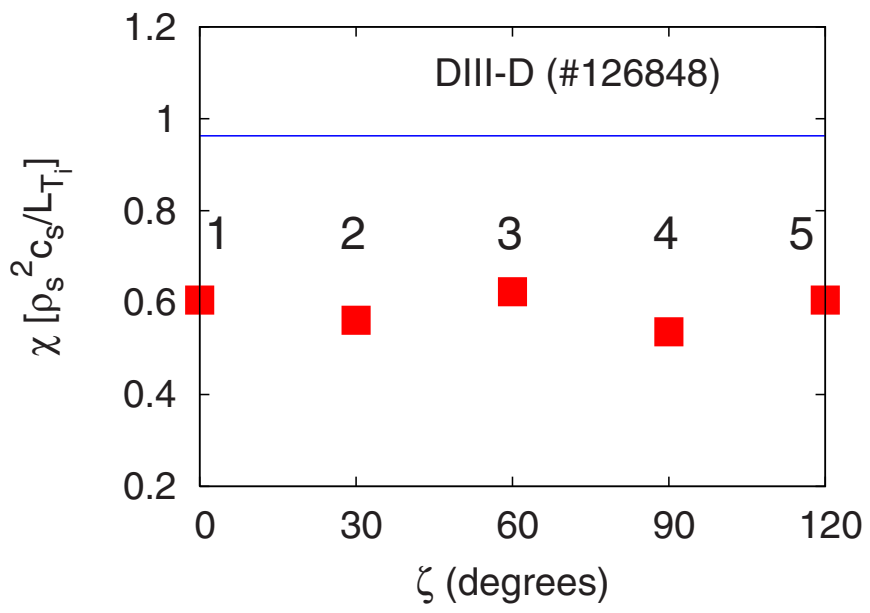

FIG. 7. (Color online) Comparison of the anomalous ion heat diffusivity between NCSX (boxes) and DIII-D (line). For the stellarator case, we select five different flux tubes along the toroidal Boozer angle, the first being identical to the fifth, in view of the threefold toroidal symmetry.

and performing a toroidal scan. This is a necessary procedure since in the stellarator configuration, one should keep in mind that the flux tubes are not necessarily equivalent. In particular, we select four different flux tubes, ranging in the toroidal period $[0,2 \pi / 3]$. The second case is a DIII-D equilibrium, corresponding to discharge No. 126848. Here, we select a surface in about the same radial position and, in view of axisymmetry, simulate only one tube. For both cases a value for the ITG about 1.5 times higher than the linear threshold (which is roughly the same for both) is recruited, while we consider a flat density profile. The result, presented in Fig. 7, leads to the following remarks.

(1) For NCSX, only a small variation of the ion diffusivity for the various tubes appears. Therefore, for such configurations, at least for this physics scenario, detailed surface scans seem to be superfluous.

(2) The comparison suggests that ITG turbulence levels (with adiabatic electrons) for NCSX will lie in the same ballpark as for a tokamak. This finding is not too surprising since to date no optimization technique engulfs the major issue of microturbulence regulation. At the same time, it is a positive sign that the relatively small minor radius of such devices (e.g., in the banana plane) does not have a dramatic negative impact. This effect should probably be attributed to the global/local shear properties, but further investigations in this direction are required.

\section{CONCLUSIONS AND OUTLOOK}

We reported on the construction of the code package GENE/GIST, which is able to conduct microturbulence studies in both stellarator and tokamak geometries, based on realistic equilibria. The required geometric elements for the gyrokinetic solver were derived from first principles, based on two independent approaches. Several numerical evaluation tests 
guarantee the reliability of the geometry interface, especially in the context of stellarator devices.

A first application was presented, by performing a crossmachine comparison for ITG turbulence, considering an adiabatic response for the electrons. We were able to discern similar heat diffusivity levels between the stellarator NCSX and the tokamak DIII-D as well as the relative insensitivity of the turbulence on the quasisymmetric geometry. The present example, of course, scratches only the surface of the many open issues (e.g., behavior of $\chi_{i}$-scaling, role of global and local shear, zonal flow response) which remain to be resolved. GENE/GIST can already perform more demanding physics, with the inclusion of kinetic electrons, impurities, and electromagnetic effects. Further, especially for stellarators, more concrete conclusions will be drawn by employing a global approach (full surface/full radius), a task which is currently under development.

\section{ACKNOWLEDGMENTS}

The authors are grateful to T. Rhodes for providing the DIII-D equilibrium data and G. Hammett for insightful discussions.

${ }^{1}$ T.-H. Watanabe, H. Sugama, and S. Ferrando-Margalet, Phys. Rev. Lett. 100, 195002 (2008).

${ }^{2}$ H. Sugama, T.-H. Watanabe, and S. Ferrando-Margalet, J. Plasma Fusion Res. 3, 041 (2008).

${ }^{3}$ O. Motojima, N. Ohyabu, A. Komori, O. Kaneko, H. Yamada, K. Kawahata, Y. Nakamura, K. Ida, T. Akiyama, N. Ashikawa, W. A. Cooper, A. Ejiri, M. Emoto, N. Ezumi, H. Funaba, A. Fukuyama, P. Goncharov, M. Goto, H. Idei, K. Ikeda, S. Inagaki, M. Isobe, S. Kado, H. Kawazome, K. Khlopenkov, T. Kobuchi, K. Kondo, A. Kostrioukov, S. Kubo, R. Kumazawa, Y. Liang, J. F. Lyon, A. Mase, S. Masuzaki, T. Minami, J. Miyazawa, T. Morisaki, S. Morita, S. Murakami, S. Muto, T. Mutoh, K. Nagaoka, Y. Nagayama, N. Nakajima, K. Nakamura, H. Nakanishi, K. Narihara, Y. Narushima, K. Nishimura, N. Nishino, N. Noda, T. Notake, H. Nozato, S. Ohdachi, Y. Oka, H. Okada, S. Okamura, M. Osakabe, T. Ozaki, B. J. Peterson, A. Sagara, T. Saida, K. Saito, S. Sakakibara, M. Sakamoto, R. Sakamoto, M. Sasao, K. Sato, M. Sato, T. Seki, T. Shimozuma, M. Shoji, H. Suzuki, Y. Takeiri, N. Takeuchi, N. Tamura, K. Tanaka, M. Y. Tanaka, Y. Teramichi, K. Toi, T. Tokuzawa, Y. Tomota, Y. Torii, K. Tsumori, K. Y. Watanabe, T. Watari, Y. Xu, I. Yamada, S. Yamamoto, T. Yamamoto, M. Yokoyama, S. Yoshimura, Y. Yoshimura, M. Yoshinuma, N. Asakura, T. Fujita, T. Fukuda, T. Hatae, S. Higashijima, A. Isayama, Y. Kamada, H. Kubo, Y. Kusama, Y. Miura, T. Nakano, H. Ninomiya, T. Oikawa, N. Oyama, Y. Sakamoto, K. Shinohara, T. Suzuki, H. Takenaga, K. Ushigusa, T. Hino, M. Ichimura, Y. Takase, F. Sano, H. Zushi, T. Satow, S. Imagawa, T. Mito, I. Ohtake, T. Uda, K. Itoh, K.
Ohkubo, S. Sudo, K. Yamazaki, K. Matsuoka, Y. Hamada, and M. Fujiwara, Nucl. Fusion 43, 1674 (2003).

${ }^{4}$ G. Grieger, C. Beidler, E. Harmeyer, J. Junker, J. Kisslinger, W. Lotz, P. Merkel, A. Montvai, J. Nührenberg, F. Rau, A. Schlüter, H. Wobig, and R. Zille, Plasma Physics and Controlled Nuclear Fusion Research, Proceedings of the 12th International Conference, Nice, 1988 (IAEA, Vienna, 1999), Vol. 2, p. 369.

${ }^{5}$ P. Xanthopoulos, F. Merz, T. Görler, and F. Jenko, Phys. Rev. Lett. 99, 035002 (2007).

${ }^{6}$ F. Jenko, W. Dorland, M. Kotschenreuther, and B. N. Rogers, Phys. Plasmas 7, 1904 (2000)

${ }^{7}$ T. Dannert and F. Jenko, Phys. Plasmas 12, 072309 (2005).

${ }^{8}$ F. Merz, Ph.D. thesis, University of Münster, 2008.

${ }^{9}$ S. P. Hirshman, W. I. van Rij, and P. Merkel, Comput. Phys. Commun. 43, 143 (1986)

${ }^{10}$ A. H. Boozer, Phys. Fluids 23, 2283 (1980).

${ }^{11}$ D. Told, F. Jenko, P. Xanthopoulos, L. D. Horton, E. Wolfrum, and ASDEX Upgrade Team, Phys. Plasmas 15, 102306 (2008).

${ }^{12}$ W. A. Cooper, Plasma Phys. Controlled Fusion 34, 1011 (1992).

${ }^{13}$ A. Reiman, L. Ku, D. Monticello, S. Hirschman, S. Hudson, C. Kessel, E. Lazarus, D. Mikkelsen, M. Zarnstorff, L. A. Berry, A. Boozer, A. Brooks, W. A. Cooper, M. Drevlak, E. Fredrickson, G. Fu, R. Goldston, R. Hatcher, M. Isaev, C. Jun, S. Knowlton, J. Lewandowski, Z. Lin, J. F. Lyon, P. Merkel, M. Mikhailov, W. Miner, H. Mynick, G. Neilson, B. E. Nelson, C. Nuehrenberg, N. Pomphrey, M. Redi, W. Reiersen, P. Rutherford, R. Sanchez, J. Schmidt, D. Spong, D. Strickler, A. Subbotin, P. Valanju, and R. White, Phys. Plasmas 8, 2083 (2001).

${ }^{14}$ J. L. Luxon, R. Anderson, F. Batty, C. B. Bramson, N. H. Brooks, B. Brown, B. Burley, K. H. Burrell, R. Callis, G. Campbell, T. N. Carlstrom, A. P. Colleraine, J. Cummings, L. Davis, J. C. DeBoo, S. Ejima, R. Evanko, H. Fukumoto, R. Gallix, J. Gilleland, T. Glad, P. Gohil, A. Gootgeld, R. J. Groebner, S. Hanai, J. Haskovec, E. Heckman, M. Heilberger, F. J. Helton, N. Hosogane, C.-L. Hsieh, G. L. Jackson, G. Jahns, G. Janeschitz, E. Johnson, A. G. Kellman, J. S. Kim, J. Kohli, A. Langhorn, L. L. Lao, P. Leek, S. Lightner, J. Lohr, M. A. Mahdavi, M. Mayberry, B. McHarg, T. McKelvey, R. Miller, C. P. Moeller, D. Moore, A. Nerem, P. Noll, T. Okhawa, N. Ohyabu, T. H. Osborne, D. O. Overskei, P. I. Petersen, T. W. Petrie, J. Phillips, R. Prater, J. Rawls, E. E. Reis, D. Remsen, P. Riedy, P. Rock, K. Schaubel, D. P. Schissel, J. T. Scoville, R. Seraydarian, M. Shimada, T. Shoji, B. Sleaford, J. P. Smith, Jr., T. Smith, R. T. Snider, R. D. Stambaugh, R. Stav, H. St. John, R. E. Stockdale, E. J. Strait, T. Stree, T. S. Taylor, J. Tooker, M. Tupper, S. K. Wong, and S. Yamakuchi, Plasma Physics and Controlled Nuclear Fusion Research, 1986 (IAEA, Vienna, 1987), Vol. I, p. 159.

${ }^{15}$ D. V. Anderson, W. A. Cooper, R. Gruber, S. Merazzi, and U. Schwenn, Int. J. High Perform. Comput. Appl. 4, 34 (1990).

${ }^{16}$ M. A. Beer, S. C. Cowley, and G. W. Hammett, Phys. Plasmas 2, 2687 (1995).

${ }^{17}$ W. D. D'Haeseleer, W. N. G. Hitchon, J. D. Callen, and J. L. Shohet, Flux Coordinates and Magnetic Field Structure (Springer, Berlin, 1991).

${ }^{18}$ P. Xanthopoulos and F. Jenko, Phys. Plasmas 13, 092301 (2006).

${ }^{19}$ S. R. Hudson, Phys. Plasmas 13, 042511 (2006).

${ }^{20}$ J. L. Johnson and J. M. Greene, Plasma Phys. 9, 611 (1967). 